\title{
Fasting glucose variability in young adulthood and incident diabetes, cardiovascular disease and all-cause mortality
}

\author{
Michael P. Bancks ${ }^{1}$ (D) April P. Carson ${ }^{2} \cdot$ Cora E. Lewis $^{2} \cdot$ Erica P. Gunderson $^{3} \cdot$ Jared P. Reis $^{4} \cdot$ Pamela J. Schreiner $^{5}$. \\ Yuichiro Yano $^{6} \cdot$ Mercedes R. Carnethon $^{7}$
}

Received: 3 January 2019 / Accepted: 15 April 2019 / Published online: 22 May 2019

(C) Springer-Verlag GmbH Germany, part of Springer Nature 2019

\begin{abstract}
Aims/hypothesis The aim of this study was to determine whether long-term intra-individual variability in fasting glucose (FG) during young adulthood is associated with incident diabetes, cardiovascular disease (CVD) and mortality.

Methods We included participants from the Coronary Artery Risk Development in Young Adults (CARDIA) study, ages 1830 years at baseline (1985-1986) and followed with eight examinations for up to 30 years. Long-term glucose variability was assessed using the $\mathrm{CV}(\mathrm{CV}-\mathrm{FG})$ and the absolute difference between successive FG measurements (average real variability; ARVFG). For participants who developed any event (diabetes, CVD or mortality), FG variability measurement was censored at the examination prior to event ascertainment. We estimated HRs for incident diabetes, CVD and mortality with adjustment for demographics, baseline FG, change in FG (censor - baseline) and time-varying education, smoking, alcohol consumption, BMI, physical activity, systolic BP, BP medications, LDL-cholesterol and cholesterol medications (and incident diabetes and diabetes medications for CVD and mortality outcomes).

Results Among 3769 black and white participants, there were 317 incident diabetes cases (102,677 person-years), 159 incident CVD events (110,314 person-years) and 174 deaths (111,390 person-years). After adjustment, HRs per 1 SD higher ARV-FG were $1.64(95 \%$ CI 1.52, 1.78) for diabetes, 1.15 (95\% CI 1.01, 1.31) for CVD and 1.25 (95\% CI 1.11, 1.40) for mortality. The HRs per 1 SD higher CV-FG were 1.39 (95\% CI 1.21, 1.58) for diabetes, 1.32 (95\% CI 1.13, 1.54) for CVD and 1.08 (95\% CI $0.92,1.27)$ for mortality, after adjustment. The cause-specific HRs per 1 SD higher ARV-FG were 1.29 (95\% CI 1.14, 1.47) for non-CVD death and $1.05(95 \%$ CI $0.76,1.45)$ for CVD death. We did not observe evidence for effect modification of any association by sex or race.

Conclusions/interpretation Our results suggest that higher intra-individual FG variability during young adulthood before the onset of diabetes is associated with incident diabetes, CVD and mortality.
\end{abstract}

Keywords Cardiovascular disease $\cdot$ Epidemiology $\cdot$ Fasting blood glucose $\cdot$ Glucose variability $\cdot$ Mortality $\cdot$ Type 2 diabetes Young adulthood

Electronic supplementary material The online version of this article (https://doi.org/10.1007/s00125-019-4901-6) contains peer-reviewed but unedited supplementary material, which is available to authorised users.

Michael P. Bancks

mbancks@wakehealth.edu

Division of Public Health Sciences, Department of Epidemiology \& Prevention, Wake Forest University School of Medicine, 525 Vine Street, 5th Floor, Winston-Salem, NC 27101, USA

2 University of Alabama at Birmingham, Birmingham, AL, USA
3 Division of Research, Kaiser Permanente Northern California, Oakland, CA, USA

4 National Heart, Lung, and Blood Institute, Bethesda, MD, USA

5 University of Minnesota, Minneapolis, MN, USA

6 Duke University, Durham, NC, USA

7 Northwestern University, Chicago, IL, USA 


\section{Research in context}

\section{What is already known about this subject?}

- Blood glucose variability is present during middle and older age, particularly among individuals with diabetes

- Greater glucose variability is associated with higher risk for incident cardiovascular disease and mortality

\section{What is the key question?}

- Is variability in blood glucose level present during young adulthood before the onset of diabetes and, if so, associated with risk for diabetes, cardiovascular disease and death?

\section{What are the new findings?}

- Greater fasting glucose variability during young adulthood before the onset of diabetes was associated with greater risk for diabetes, cardiovascular disease and death during middle age

\section{How might this impact on clinical practice in the foreseeable future?}

- If further work proves variable glucose levels are a marker of future risk, healthcare providers should consider monitoring of glucose levels for individuals who experience variable glucose levels over time

$\begin{array}{ll}\text { Abbreviations } \\ \text { ARV } & \text { Average real variability } \\ \text { ARV-FG } & \text { Average real variability of fasting glucose } \\ \text { CARDIA } & \begin{array}{l}\text { Coronary Artery Risk Development in Young } \\ \text { Adults }\end{array} \\ \text { CVD } & \text { Cardiovascular disease } \\ \text { CV-FG } & \text { CV of fasting glucose } \\ \text { EU } & \text { Exercise unit } \\ \text { FG } & \text { Fasting glucose }\end{array}$

\section{Introduction}

For individuals with diabetes, prevention of complications requires maintenance of blood glucose levels within a commonly accepted range and limiting the hypo- and hyperglycaemic excursions from this range [1,2]. Recent epidemiological research suggests that greater long-term variability in traditional measures of blood or plasma glucose during middle and older age is associated with higher risk for cardiovascular disease (CVD) events and mortality among adults with diabetes [3-14]. However, among adults without diabetes at study initiation, studies that have reported an association between glucose variability and outcomes have not taken into account subsequent development of diabetes and diabetes medication use. Furthermore, blood glucose tends to increase over the lifespan and it is important to distinguish long-term variability in glucose from increases in glucose that occur with aging and in association with aging-related comorbidities.

The Coronary Artery Risk Development in Young Adults (CARDIA) study is an ideal setting to determine the natural variability of glucose over 30 years beginning during young adulthood in a population followed into middle age for incident diabetes, CVD events and mortality. Our objective was to assess whether variability in fasting glucose (FG) during young adulthood is associated with incident diabetes, CVD and mortality. We hypothesised that higher variability of FG during young adulthood would be associated with higher risk for incident diabetes, CVD and mortality as compared with lower FG variability, independent of increases in glucose over time.

\section{Methods}

Study population CARDIA is a longitudinal observational study of individuals recruited from four US metropolitan communities. Details regarding the CARDIA study design have been published previously [15]. Briefly, 5115 black and white men and women aged 18-30 years who were free from CVD were enrolled in 1985-1986 [15]. Participants were invited to participate in follow-up examinations $2,5,7,10,15,20,25$ and 30 (2015-2016) years after baseline. Participation rates at these examinations were high: $91 \%, 86 \%, 81 \%, 79 \%, 74 \%$, $72 \%, 72 \%$ and $71 \%$, respectively, of the surviving cohort. Written informed consent was obtained at enrolment and each subsequent examination. Institutional review boards at each study site and coordinating centre have granted approval for all examinations and contacts.

Structured questionnaires were used at each clinic visit to collect information on participant demographics, medical history and use of medications, and health behaviours, such as past and current use of tobacco, regular alcohol consumption and leisure-time physical activity $[15,16]$. CARDIA researchers derived an overall physical activity score in 
'exercise units' (EU), with a score of 300 EU corresponding to 30 min of moderate physical activity, five times per week. [17] Prior to examination year 20, seated BP was measured in triplicate after 5 min of rest using a random-zero sphygmomanometer. At year 20 and later, BP was measured using an automated oscillometric BP monitor (Omron HEM-907XL; Online Fitness, Santa Monica, CA, USA) with values standardised across examinations to the sphygmomanometric measures; systolic and diastolic BP were determined as the average of the last two measurements [18]. Body weight was measured with a calibrated balance beam scale and height was measured with a vertical ruler. BMI was calculated as weight in kilograms divided by height in meters squared. At each examination, blood was drawn by venipuncture and serum separation was performed before aliquots were stored at $-70^{\circ} \mathrm{C}$ and shipped on dry ice to central laboratories. Total cholesterol and triacylglycerols were measured enzymatically within 6 weeks of collection, HDL-cholesterol was determined by precipitation with dextran sulphate-magnesium chloride and LDL-cholesterol was determined by the Friedewald equation [19-21].

FG and incident diabetes FG was determined in non-pregnant individuals who reported fasting $\geq 8 \mathrm{~h}$ at baseline and $7,10,15$, 20, 25 and 30 years after baseline. At baseline, serum glucose was measured using the hexokinase ultraviolet method by American Bio-Science Laboratories (Van Nuys, CA, USA). At examination years 7-20, serum glucose was measured using hexokinase coupled to glucose 6-phosphate dehydrogenase by Linco Research (St Louis, MO, USA). Quality control was performed using a commercially purchased pool of controls; within-run precision was below $1 \% \mathrm{CV}$ and between-run precision was below $2 \%$. Serum glucose was measured at years 25 and 30 at the Collaborative Studies Clinical Laboratory at the University of Minnesota. Test-retest correlation between the year 25 and 30 assays was 0.988 . The data used herein employ findings from a recalibration study, performed to harmonise glucose values across CARDIA examinations [22]. Diabetes was determined at each examination using a combination of available information on $\mathrm{FG} \geq 7.0 \mathrm{mmol} / 1$ (examination years 0 and 7-30), $2 \mathrm{~h} 75 \mathrm{~g}$ OGTT $\geq 11.1 \mathrm{mmol} / 1$ (examination years 10,20 and 25 ), nonfasting glucose $\geq 11.1 \mathrm{mmol} / 1$ (examination years 0 and 7-30), $\mathrm{HbA}_{1 \mathrm{c}} \geq 48 \mathrm{mmol} / \mathrm{mol}(\geq 6.5 \%$ ) (examination years 20 and 25 ) and use of diabetes medications (all examinations), previously assessed in CARDIA [23].

CVD events and mortality During study examinations and annual contacts completed via mail, phone or email using standardised questionnaires, participants or designated proxies were queried about overnight hospital admissions, outpatient procedures and deaths that occurred since the last study contact. Additional semi-annual contacts were conducted, also via mail, phone or email, and were designed to provide research staff with the opportunity to update participants' vital status and contact information. Medical records were requested for potential cardiovascular hospitalisations and outpatient procedures. Vital status was also obtained through periodic surveillance of the National Death Index. Trained physicians using detailed event definitions reviewed medical records, autopsy reports, death certificates, informant interviews and other evidence, and adjudicated cases independently in pairs. In the instance of disagreement in initial event adjudication, the case was reviewed by the full CARDIA Endpoints Committee. The primary CVD outcome was the first occurrence of any fatal or non-fatal CVD event, including myocardial infarction, non-myocardial infarction acute coronary syndrome, coronary revascularisation, stroke, transient ischaemic attack, hospitalisation for acute decompensated heart failure, intervention for peripheral arterial disease or underlying cause of death due to CVD. We recorded incident CVD events and mortality that occurred between the baseline examination and 31 August 2016.

Statistical analysis We excluded one person who withdrew consent during follow-up and 1345 individuals who had fewer than three FG measures. We calculated two measures of intraindividual FG variability for each participant: $\mathrm{CV}$ (CV-FG) and average real variability (ARV; ARV-FG). CV-FG (\%) was calculated for each individual as the SD of FG divided by mean FG, and then divided by the square root of the ratio of FG measurements $(n)$ to $n-1, \sqrt{n /(n-1)}$, to account for the possible influence on $\mathrm{CV}$ of the FG measurement number [24]. ARV has been previously modelled for BP in CARDIA [25]. Here, ARV-FG (mmol/l per year) was calculated as the absolute difference between successive measurements of FG, divided by the duration between FG measurements, to create annualised ARV-FG between examinations. We summed over examinations and divided by the number of years contributing to ARV-FG determination, to account for variable duration of ARV-FG. To minimise the potential for diabetes medications or clinical CVD events to influence glucose variability determination, FG values measured at or after examinations where identification of diabetes or a CVD event occurred did not contribute to CV-FG or ARV-FG. This novel methodological approach is in contrast to the prior research on this topic, where glucose measures with concomitant medication use contributed to variability $[26,27]$. Change in FG was determined as the difference in FG at the last examination for FG variability measurement before censoring and baseline FG (i.e., last FG value minus baseline FG).

Participants who missed an examination were encouraged to participate in subsequent examination years, but missing data are mostly due to participants not returning and are not missing at random. Missing covariate data were generated 
using two-step fully conditional specification imputation methods $[28,29]$. The proportion of observations missing data before imputation was largest for LDL-cholesterol, ranging from $8 \%$ to $17 \%$ over all examinations, and less than $10 \%$ for all other covariates at each examination.

We used extended Cox proportional hazards regression to estimate the association of a $1 \mathrm{SD}$ increment for continuous ARV-FG and CV-FG with incident diabetes, CVD and allcause mortality. Our modelling approach included adjustment for age, sex, race, field centre, baseline FG value, change in FG and time-varying data for educational attainment, smoking status, regular alcohol consumption, BMI, physical activity, systolic BP, use of BP-lowering medication, LDLcholesterol and use of cholesterol-lowering medication. For the outcomes of CVD and mortality, we included timevarying incident diabetes and use of diabetes medication in the adjustment scheme. Person-time at risk for each respective outcome started at baseline and ended at the examination at which the respective event was ascertained, date of respective event from medical/death records or date of last contact. We assessed for collinearity between adjustment variables by calculating variance inflation factors and did not find evidence for collinearity; all variance inflation factors were $<1.6$. We assessed the sensitivity of our estimates (1) when excluding individuals with missing baseline covariate data, without imputing missing data and with carrying forward prior values; (2) when censoring individuals who developed diabetes during follow-up and treating this as a competing risk for CVD and mortality outcomes; and (3) when restricting the sample to individuals who had FG values at each of the first four glucose assessments. To better understand the association between FG variability and cause-specific death, we assessed the HRs for CVD-related deaths and non-CVD deaths taking into account competing risk of the alternative cause of death [30]. We

Table 1 Characteristics at baseline for 3769 participants by quartile of ARV in FG from 1985-1986 to 2010-2011

\begin{tabular}{|c|c|c|c|c|c|}
\hline \multirow[t]{2}{*}{ Characteristic } & \multicolumn{5}{|l|}{$\mathrm{ARV}$ in $\mathrm{FG}$} \\
\hline & Quartile 1 & Quartile 2 & Quartile 3 & Quartile 4 & $p$ value $^{\mathrm{a}}$ \\
\hline ARV glucose, $\mathrm{mmol} / \mathrm{l}$ per year, range & $0.0001,0.008$ & $0.009,0.012$ & $0.013,0.018$ & $0.019,0.08$ & \\
\hline$n$ & 942 & 942 & 943 & 942 & \\
\hline Age, years & $24.7 \pm 3.7$ & $25.3 \pm 3.5$ & $24.9 \pm 3.6$ & $25.1 \pm 3.6$ & $<0.005$ \\
\hline Women & $560(59)$ & $542(58)$ & $542(57)$ & $463(49)$ & $<0.001$ \\
\hline Black & $470(50)$ & $398(42)$ & 409 (43) & $499(53)$ & $<0.001$ \\
\hline Education, $>4$ years college & $91(10)$ & $133(14)$ & $105(11)$ & $70(7)$ & $<0.001$ \\
\hline Current smoker & $247(26)$ & $240(25)$ & $250(27)$ & $301(32)$ & $<0.05$ \\
\hline No daily alcohol & $389(41)$ & $354(38)$ & $371(39)$ & $352(37)$ & 0.53 \\
\hline BMI, $\mathrm{kg} / \mathrm{m}^{2}$ & $24.1 \pm 5.0$ & $23.9 \pm 4.3$ & $24.1 \pm 4.3$ & $24.8 \pm 5.2$ & $<0.001$ \\
\hline Systolic BP, mmHg & $109.6 \pm 10.9$ & $109.7 \pm 10.8$ & $109.5 \pm 10.3$ & $111.1 \pm 10.8$ & $<0.005$ \\
\hline Diastolic BP, mmHg & $68.4 \pm 9.2$ & $68.5 \pm 9.0$ & $68.0 \pm 9.7$ & $68.8 \pm 9.9$ & 0.34 \\
\hline BP-lowering medication use & $29(3)$ & $9(1)$ & $20(2)$ & $18(2)$ & $<0.05$ \\
\hline Physical activity, EU & $429 \pm 305$ & $408 \pm 298$ & $424 \pm 289$ & $419 \pm 296$ & 0.48 \\
\hline LDL-cholesterol, mmol/1 & $2.81 \pm 0.8$ & $2.86 \pm 0.8$ & $2.83 \pm 0.76$ & $2.81 \pm 0.82$ & 0.50 \\
\hline FG at baseline, $\mathrm{mmol} / \mathrm{l}$ & $4.57 \pm 0.38$ & $4.54 \pm 0.43$ & $4.51 \pm 0.44$ & $4.50 \pm 0.52$ & $<0.05$ \\
\hline FG at variability censoring examination, $\mathrm{mmol} / \mathrm{l}$ & $5.13 \pm 0.43$ & $5.22 \pm 0.51$ & $5.27 \pm 0.57$ & $5.40 \pm 0.66$ & $<0.001$ \\
\hline Weighted average FG, mmol/1 & $4.93 \pm 0.32$ & $4.98 \pm 0.33$ & $5 \pm 0.34$ & $5.08 \pm 0.38$ & $<0.001$ \\
\hline Incident diabetes before event of administrative censoring & $39(4)$ & $46(5)$ & $67(7)$ & $165(18)$ & $<0.001$ \\
\hline Number of glucose measurements ${ }^{\mathrm{b}}$ & $4.7 \pm 1.3$ & $5.6 \pm 1.3$ & $5.9 \pm 1.3$ & $5.5 \pm 1.5$ & $<0.001$ \\
\hline CV glucose, $\%$ & $5.7 \pm 2.6$ & $7.0 \pm 2.6$ & $7.7 \pm 2.4$ & $10.0 \pm 3.2$ & $<0.001$ \\
\hline ARV glucose, $\mathrm{mmol} / \mathrm{l}$ per year & $0.006 \pm 0.002$ & $0.01 \pm 0.001$ & $0.015 \pm 0.002$ & $0.025 \pm 0.008$ & $<0.001$ \\
\hline Proportion of ARV in a positive direction, $\%$ & $0.75 \pm 0.24$ & $0.70 \pm 0.20$ & $0.66 \pm 0.18$ & $0.63 \pm 0.19$ & $<0.001$ \\
\hline Have the majority of ARV in a positive direction & $799(85)$ & $785(83)$ & $793(84)$ & $727(77)$ & $<0.001$ \\
\hline
\end{tabular}

Data are mean $\pm \mathrm{SD}$ or $n(\%)$ unless stated otherwise

ARV in FG was calculated as the absolute difference between successive measurements of FG, divided by the duration between FG measurements, to create annualised ARV-FG between examinations. Annualised ARV-FG was then summed over examinations and divided by the number of years contributing to ARV-FG determination

${ }^{\mathrm{a}} p$ value for global test: ANOVA for continuous variables and Pearson $\chi^{2}$ tests for categorical variables

${ }^{\mathrm{b}}$ Range $n: 3-7$ 
assessed effect measure modification of each association by race and sex. SAS software version 9.4 (SAS Institute, Cary NC) was used for all analyses.

\section{Results}

Among 3769 CARDIA participants initially free from CVD, the mean (SD) age at enrolment was 25.0 (3.6) years, $47 \%$ were African-American and $56 \%$ were women. Table 1 presents participant characteristics according to quartile of ARVFG only, as characteristics were generally similar for CV-FG quartiles (electronic supplementary material [ESM] Table 1). Current smoking at enrolment was associated with greater subsequent variability for both ARV-FG and CV-FG. For both ARV-FG and CV-FG, the incidence of diabetes over followup was highest among the top quartile of variability. Older age, male sex and greater BMI, SBP and number of glucose measurements were all associated with greater ARV-FG over follow-up, but not with CV-FG. Lower percentage of variability in the positive direction was associated with greater ARVFG over follow-up.

Over a median follow-up of 29.8 years, there were 317 incident diabetes cases (102,677 person-years), 159 incident CVD events (110,314 person-years) and 174 deaths from all causes (111,390 person-years). In an unadjusted model, the HR for incident diabetes per $1 \mathrm{SD}$ higher ARV-FG was 1.78 (95\% CI 1.66, 1.91) (Fig. 1a). After adjustment for age, sex, race, field centre, baseline $\mathrm{FG}$, and change in FG and timevarying risk factors, the diabetes HR per $1 \mathrm{SD}$ higher ARV-FG was $1.64(1.52,1.78)$. The association between 1 SD higher $\mathrm{CV}-\mathrm{FG}$ and incident diabetes was similar in direction and lower in magnitude for each model (unadjusted HR 1.50; 95\% CI 1.35, 1.66 and adjusted HR 1.39; 95\% CI 1.21, 1.58) (Fig. 1b).

Before adjustment, greater ARV-FG was associated with greater HRs for incident CVD (Fig. 1a). The HR for incident CVD per 1 SD higher ARV-FG was 1.34 (95\% CI 1.17, 1.52). The HR for CVD after adjustment for age, sex, race, field centre, baseline FG and change in FG per $1 \mathrm{SD}$ higher ARVFG was $1.25(95 \%$ CI 1.10, 1.42) and this association remained after adjustment for time-varying CVD risk factors. The association between CV-FG and incident CVD was similar in magnitude and direction for each model (Fig. 1b).

In an unadjusted model, $1 \mathrm{SD}$ higher ARV-FG was associated with $37 \%$ higher HR $(95 \%$ CI 1.22, 1.55) for all-cause mortality (Fig. 1a). These HRs were slightly attenuated after adjustment for basic demographics, baseline FG and change in glucose and after further adjustment for time-varying CVD risk factors, but remained significantly associated with allcause mortality (HR 1.25; 95\% CI 1.11, 1.40). The association between $1 \mathrm{SD}$ higher $\mathrm{CV}-\mathrm{FG}$ and mortality was similar in direction, but not strong in magnitude (Fig. 1b). Of the 174

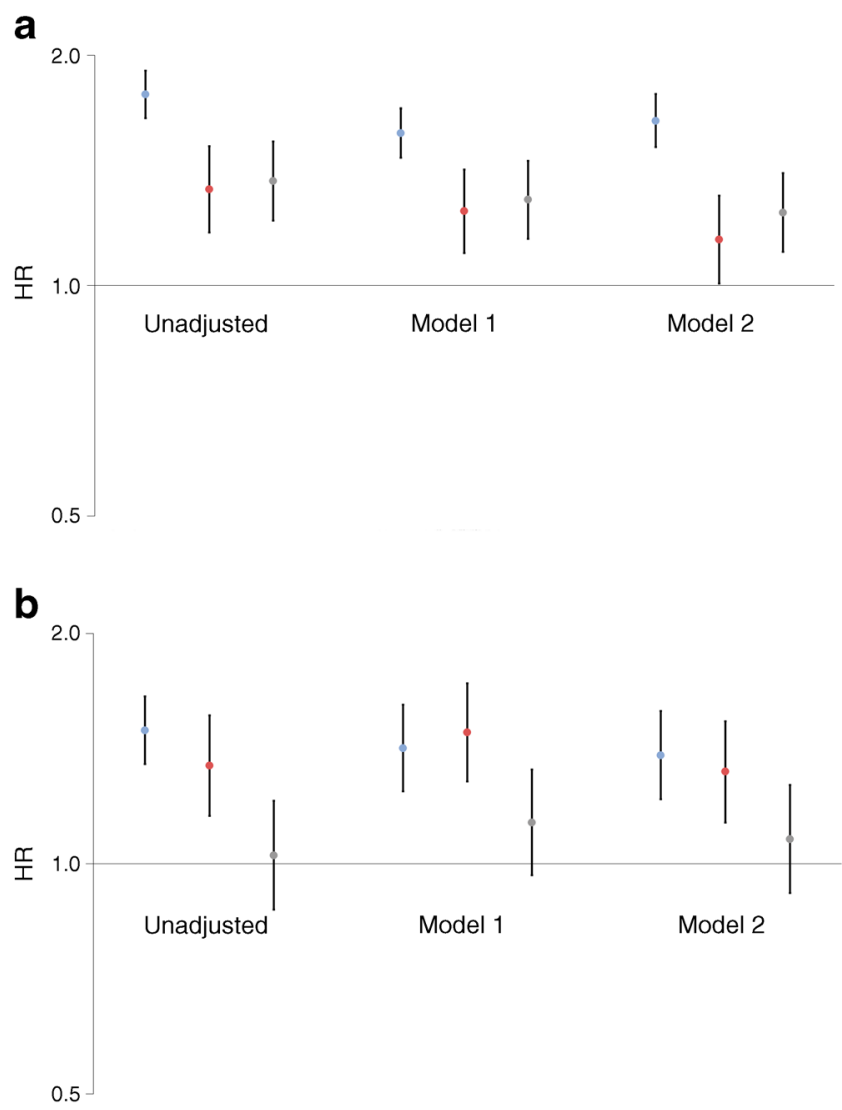

Fig. 1 Multivariable adjusted HRs (95\% CIs) for incident diabetes (blue marker), CVD (red marker) and all-cause mortality (grey marker) according to $1 \mathrm{SD}$ higher (a) ARV (1 SD: $0.009 \mathrm{mmol} / \mathrm{l}$ per year) in FG and (b) CV (1 SD: $3.4 \%$ ) in FG. Model 1 is adjusted for age, sex, race, field centre, baseline FG and change in FG from baseline to the examination of last glucose variability measurement. Model 2 includes the variables in model 1 and further adjustment for time-varying educational attainment, smoking status, alcohol consumption, physical activity. BMI, systolic BP, BP-lowering medication use, LDL-cholesterol and cholesterol-lowering medication use. For CVD and mortality outcomes, model 2 also includes adjustment for time-varying incidence of diabetes and diabetes medication use

deaths, 29 were classified as CVD mortality. Deaths due to any cancer $(n=34)$ were the most frequent non-CVD causes. The association between ARV-FG and overall mortality was mainly due to non-CVD deaths. The cause-specific HRs per 1 SD higher ARV-FG were 1.29 (95\% CI 1.14, 1.47) for nonCVD death and 1.05 (95\% CI 0.76, 1.45) for CVD death. HRs from the subdistribution model taking into account competing risk of death were similar in magnitude and direction to the cause-specific HRs.

When assessing each association according to quartile of ARV-FG, we observed a potential dose-response for ARV-FG quartile with hazards for diabetes (Fig. 2). We observed a possible threshold effect wherein HRs were substantively higher for the highest quartile, but not the interquartile range, compared with the lowest quartile for both incident CVD and mortality. We did not observe evidence for effect modification on the multiplicative scale of any association by sex or race 


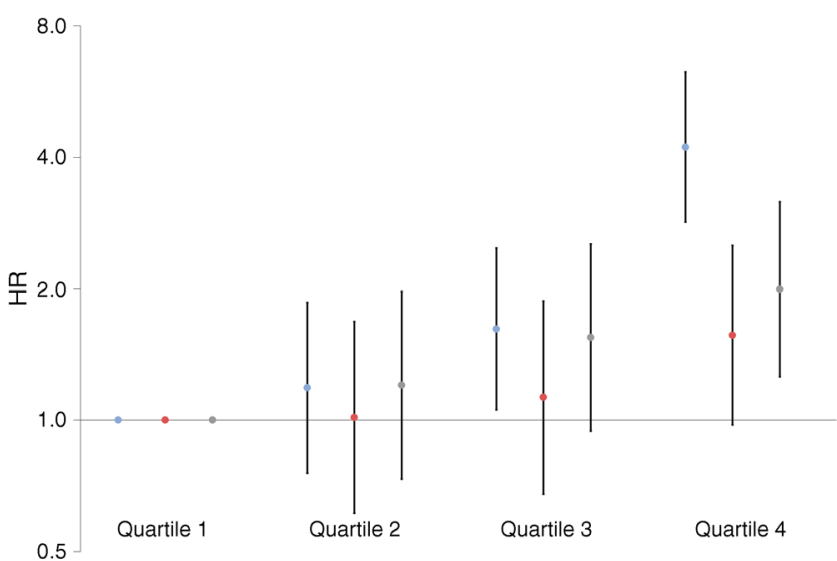

Fig. 2 Multivariable adjusted HRs (95\% CIs) for incident diabetes (blue marker), CVD (red marker) and all-cause mortality (grey marker) according to quartile of ARV in FG. HRs are adjusted for age, sex, race, field centre, baseline FG, change in FG from baseline to the examination of last glucose variability measurement and time-varying educational attainment, smoking status, alcohol consumption, physical activity, BMI, systolic BP, BP-lowering medication use, LDL-cholesterol and cholesterollowering medication use. For CVD and mortality outcomes, model 2 also includes adjustment for time-varying incidence of diabetes and diabetes medication use

(all $p$ for interaction $>0.05$ ). Estimates from non-imputed data and when censoring individuals at the time of diabetes development during follow-up were similar in strength and direction of association to the primary findings (ESM Tables 2 and 3). When the sample was restricted to individuals who had FG values at each of the first four glucose assessments, we observed negligible attenuation in the HRs for each association, with the exception of the association between CV-FG and allcause mortality where we observed a strengthening of the association (ESM Table 4).

\section{Discussion}

In this prospective cohort of adults followed for 30 years, greater intra-individual variability in FG during young adulthood was associated with higher risk for incident diabetes, CVD and all-cause mortality by middle age. Importantly, glucose variability was assessed before the onset of diabetes, measured by both absolute average difference between successive measurements and as a percentage of mean exposure, and was consistently associated with incident diabetes, CVD and mortality. Both measures of glucose variability were associated with incident diabetes, CVD and mortality after adjustment for baseline glucose level, change in glucose over follow-up, and time-varying changes in traditional diabetes and CVD risk factors, including the development of diabetes and diabetes medication use after the assessment of glucose variability for CVD and mortality outcomes. Furthermore, the findings for CVD and all-cause mortality were consistently observed with multiple approaches for sensitivity analyses to address the development of diabetes over follow-up. Together, these results suggest that higher glucose variability before the onset of diabetes may be a harbinger of serious health outcomes.

Our findings of an association of long-term glycaemic variability with CVD and mortality align with other studies on this topic [3-12, 31, 32]. A preponderance of this previous research has been focused on glycaemic variability among individuals with frank type 2 diabetes [3-7, 9, 10]. Findings from interventions among diabetes populations suggest that greater visit-to-visit variability over $2-7$ years is associated with greater risk for incident micro- and macrovascular events $[3,7]$ and mortality $[3,4]$. Our multivariable adjusted incident CVD HR estimates per $1 \mathrm{SD}$ in ARV-FG are similar to those reported from the Veteran's Affairs Diabetes Trial [7]. Our estimates per $1 \mathrm{SD}$ in ARV-FG, but not CV-FG, with incident CVD and mortality were similar to those observed in the Action in Diabetes and Vascular Disease: Preterax and Diamicron Modified Release Controlled Evaluation Trial [3]. The unadjusted and adjusted estimates for the association per $1 \mathrm{SD}$ CV-FG with incident diabetes observed here in CARDIA are higher compared with those reported from a study population drawn from the Korean National Health Insurance System [31]. This study included an older population at baseline, shorter follow-up, annual FG assessment and a different diabetes case definition of $F G \geq 7.0 \mathrm{mmol} / 1$ or an insurance claim for diabetes medication use, compared with our current work. In contrast to our findings, Ghouse et al did not observe an association between glucose variability and incident diabetes using a cohort drawn from the Copenhagen (Denmark) General Practitioner's Laboratory [32]. This work by Ghouse et al differed methodologically from our work and included an older study population, shorter follow-up, three annual $\mathrm{HbA}_{1 \mathrm{c}}$ measurements and a diabetes case definition of discharge diagnosis of type 2 diabetes or treatment with oral glucose-lowering drugs. Investigators of the Kailuan study, a large population-based cohort in China, reported an association of CV-FG with incident CVD and mortality [8]. They observed a potential threshold effect for higher risk limited to the top quartile of variability and most pronounced among individuals who did not have diabetes [8]. In our current study, we focused on assessing glucose variation before the onset of diabetes in relation to incident CVD and mortality and we adjusted for the subsequent development of diabetes. Our approach is a methodological strength because we only included FG measurements prior to diabetes onset in our glucose variability measures to reduce the potential for residual confounding due to the initiation of glucose-lowering medications. Diabetes medication use disrupts the natural course of glucose variability and may alter any association of glucose variability with incident CVD and mortality.

We were able to extend prior work in this area on the causes of death associated with higher glucose variability. In our 
study, over $80 \%$ of deaths were due to non-CVD causes; glucose variability was associated with higher risk for incident CVD events and for non-CVD mortality, but not with CVD mortality. One potential explanation is that a non-fatal CVD event may result in clinical intervention of CVD risk factors and secondary prevention of CVD mortality. Glucose variability was not associated with CVD mortality, but we had a small number of events. The association we observed with nonCVD mortality of various origins suggests that glucose variability may be marker for underlying poor health.

There are multiple potential pathophysiological mechanisms that link long-term glucose variability with incident diabetes, CVD and mortality. Firstly, acute glucose fluctuations are shown to trigger production and release of oxidative stress isomers to a greater extent than sustained episodes of hyperglycaemia [33]. Secondly, transient hyperglycaemia may induce lasting epigenetic changes that activate inflammatory pathways [34]. Lastly, higher long-term variability may be a corollary of underlying poor health and other comorbidities. While CARDIA participants were generally healthy at enrolment, participants who had the highest variability over followup were more likely to smoke, and had higher BMI and BP at baseline. We observed slightly stronger associations of ARVFG with CVD and mortality than for CV-FG, though associations were consistently in the same direction. Different metrics of variability rely on unique statistical assumptions which may lend to stronger correlation with other statistical estimates (e.g., mean and change) and may also reflect distinct physiological conditions (e.g., direction of variability and absolute variability rather than percentage) [13]. Future research should assess the utility of multiple measures of blood or plasma glucose and variability with various morbidities and mortality.

Strengths of our study include generalisability of our findings to populations with similar demographic, health behaviour and cardiovascular risk profiles. Unlike prior studies, we characterised long-term glucose variability during young adulthood before the onset of diabetes in a biracial cohort. CARDIA employs thorough CVD event adjudication and we used rigorous statistical methods to disentangle glucose variability from bulk increases and to update time-varying CVD risk factors. Some limitations of our study merit consideration. The number of FG measurements per individual was not equal across the spectrum of glucose variability; however, the pattern of association was similar when accounting for the variable number of measurements and duration between measurements. The duration between FG measurements was 3-7 years and may not reflect short-term variability in glucose traditionally assessed among individuals with diabetes. $\mathrm{HbA}_{1 \mathrm{c}}$ and $2 \mathrm{~h}$ glucose were measured less frequently and at later points during CARDIA follow-up, preventing the assessment of variability for these measures and their association with incident CVD and mortality. It is possible that the assessment of glucose variability in this study is less sensitive than variability that incorporates information from fasting and $2 \mathrm{~h}$ glucose and $\mathrm{HbA}_{1 \mathrm{c}}$. We are unable to distinguish whether the lowering of glucose is intentional or unintentional. Among individuals with high normal glucose levels, there is unequivocal benefit from lowering glucose with intentional lifestyle and pharmacologic therapies [35, 36]. We caution not to discourage lowering high glucose with established therapies and we encourage achieving normal consistent levels of glucose. Lastly, we did not observe similar strengths in magnitude of associations between each variability measure and higher risk for mortality. It is possible that CV-FG is a less precise measurement of variability than ARV-FG, particularly at lower glucose levels where a given SD would have a greater influence on the CV-FG than a similar $\mathrm{SD}$ at higher glucose levels. With a less precise exposure and a non-specific outcome such as all-cause mortality, we would expect to observe attenuation toward the null and wider confidence limits. To that point, while our estimates per $1 \mathrm{SD}$ increment in CV-FG with risk for all-cause mortality are comparable to those of another recent study, our precision around these HR estimates is lower [37].

In this longitudinal study of black and white adults, we observed that greater glucose variability during young adulthood was associated with higher risk for incident diabetes, CVD and mortality. Associations with mortality were predominantly due to deaths from non-CVD origin. Future research should focus on understanding whether young adults experience variability in other measures of glucose such as $\mathrm{HbA}_{1 \mathrm{c}}$ and $2 \mathrm{~h}$ glucose and whether glucose variability is a corollary of other morbidity or a potential modifiable risk factor for fatal events.

Acknowledgements The authors thank the other investigators, the staff and the participants of the CARDIA study for their valuable contributions.

Data availability The datasets analysed during the current study are not publicly available due to the possibility that some information in these data might compromise research participants' privacy or consent. However, data are available from the corresponding author on request and de-identified data to the end of CARDIA examination year 25 are publicly available at: https://biolincc.nhlbi.nih.gov/studies/cardia/.

Funding MPB was partially supported by the National Heart, Lung, and Blood Institute (NHLBI) of the National Institutes of Health under Award Number T32HL069771 to conduct the current work. The Coronary Artery Risk Development in Young Adults Study (CARDIA) is conducted and supported by the NHLBI in collaboration with the University of Alabama at Birmingham (HHSN268201800005I and HHSN268201800007I), Northwestern University (HHSN268201800003I), the University of Minnesota (HHSN268201800006I) and the Kaiser Foundation Research Institute (HHSN268201800004I). The ancillary study to CARDIA (R01 DK106201, EPG, Principal Investigator) from the National Institute of Diabetes, Digestive and Kidney Diseases supported data collection for this study. The funders/sponsor of this study had no role in the design and conduct of the study; collection, management, analysis and interpretation of the data; preparation, review or approval of the manuscript; or the decision to submit the manuscript for publication. 
Duality of interest EPG receives funding unrelated to the current study from Janssen Pharmaceuticals, Inc. (June 2017). APC receives research funding unrelated to the current study from Amgen, Inc. The remaining authors declare that there is no duality of interest associated with this manuscript. The views expressed in this manuscript are those of the authors and do not necessarily represent the views of the NHLBI, the National Institutes of Health or the US Department of Health and Human Services.

Contribution statement MPB is the guarantor of this work, had full access to all of the data in the study and takes responsibility for the integrity of the data and the accuracy of the data analysis. MPB and MRC conceptualised the study and designed the analysis plan. MPB performed all statistical analyses and drafted the manuscript. MRC provided supervision to MPB. All coauthors, MPB, APC, CEL, EPG, JPR, PJS, YY and MRC, contributed to the acquisition, analysis or interpretation of data; provided critical revision of the manuscript for important intellectual content and contributed to the discussion; and approve the final version of this manuscript.

\section{References}

1. American Diabetes Association (2017) Standards of medical care in diabetes - 2017. Diabetes Care 40:S1-S135

2. Danne T, Nimri R, Battelino T et al (2017) International consensus on use of continuous glucose monitoring. Diabetes Care 40(12): 1631-1640. https://doi.org/10.2337/dc17-1600

3. Hirakawa Y, Arima H, Zoungas S et al (2014) Impact of visit-tovisit glycemic variability on the risks of macrovascular and microvascular events and all-cause mortality in type 2 diabetes: the ADVANCE trial. Diabetes Care 37(8):2359-2365. https://doi.org/ $10.2337 / \mathrm{dc} 14-0199$

4. Zinman B, Marso SP, Poulter NR et al (2018) Day-to-day fasting glycaemic variability in DEVOTE: associations with severe hypoglycaemia and cardiovascular outcomes (DEVOTE 2). Diabetologia 61(1):48-57. https://doi.org/10.1007/s00125-0174423-z

5. Xu D, Fang H, Xu W, Yan Y, Liu Y, Yao B (2016) Fasting plasma glucose variability and all-cause mortality among type 2 diabetes patients: a dynamic cohort study in Shanghai, China. Sci Rep 6(1): 39633. https://doi.org/10.1038/srep39633

6. Lin CC, Yang CP, Li CI et al (2014) Visit-to-visit variability of fasting plasma glucose as predictor of ischemic stroke: competing risk analysis in a national cohort of Taiwan Diabetes Study. BMC Med 12(1):165. https://doi.org/10.1186/s12916-014-0165-7

7. Zhou JJ, Schwenke DC, Bahn G, Reaven P (2018) Glycemic variation and cardiovascular risk in the Veteran's Affairs Diabetes Trial. Diabetes Care 41(10):2187-2194. https://doi.org/10.2337/ dc18-0548

8. Wang A, Liu X, Xu J et al (2017) Visit-to-visit variability of fasting plasma glucose and the risk of cardiovascular disease and all-cause mortality in the general population. J Am Heart Assoc 6(12): e006757. https://doi.org/10.1161/JAHA.117.006757

9. Orsi E, Solini A, Bonora E et al (2018) Haemoglobin A1c variability is a strong, independent predictor of all-cause mortality in patients with type 2 diabetes. Diabetes Obes Metab 20(8):1885-1893. https://doi.org/10.1111/dom.13306

10. Cardoso CRL, Leite NC, Moram CBM, Salles GF (2018) Longterm visit-to-visit glycemic variability as predictor of micro- and macrovascular complications in patients with type 2 diabetes: The Rio de Janeiro Type 2 Diabetes Cohort Study. Cardiovasc Diabetol 17(1):33. https://doi.org/10.1186/s12933-018-0677-0
11. Akirov A, Diker-Cohen T, Masri-Iraqi H, Shimon I (2017) High glucose variability increases mortality risk in hospitalized patients. J Clin Endocrinol Metab 102(7):2230-2241. https://doi.org/10.1210/ jc.2017-00450

12. Mendez CE, Mok KT, Ata A, Tanenberg RJ, Calles-Escandon J, Umpierrez GE (2013) Increased glycemic variability is independently associated with length of stay and mortality in noncritically ill hospitalized patients. Diabetes Care 36(12):4091-4097. https:// doi.org/10.2337/dc12-2430

13. Eslami S, Taherzadeh Z, Schultz MJ, Abu-Hanna A (2011) Glucose variability measures and their effect on mortality: a systematic review. Intensive Care Med 37(4):583-593. https://doi.org/10.1007/ s00134-010-2129-5

14. Yang YF, Li TC, Li CI et al (2015) Visit-to-visit glucose variability predicts the development of end-stage renal disease in type 2 diabetes: 10-year follow-up of Taiwan Diabetes Study. Medicine 94(44):e1804. https://doi.org/10.1097/MD.0000000000001804

15. Friedman GD, Cutter GR, Donahue RP et al (1988) CARDIA: study design, recruitment, and some characteristics of the examined subjects. J Clin Epidemiol 41(11):1105-1116. https://doi.org/10. 1016/0895-4356(88)90080-7

16. Jacobs DJ, Hahn L, Haskell W, Pirie P, Sidney S (1989) Validity and reliability of a short physical activity history: CARDIA and the Minnesota Heart Health Program. J Cardpulm Rehabil 9(11):448 459. https://doi.org/10.1097/00008483-198911000-00003

17. American College of Sports Medicine (1993) The recommended quantity and quality of exercise for developing and maintaining cardiorespiratory and muscular fitness in healthy adults. Position stand of the American College of Sports Medicine. Schweiz Z Sportmed 41:127-137

18. Hozawa A, Jacobs DR Jr, Steffes MW, Gross MD, Steffen LM, Lee DH (2009) Circulating carotenoid concentrations and incident hypertension: the Coronary Artery Risk Development in Young Adults (CARDIA) study. J Hypertens 27(2):237-242. https://doi. org/10.1097/HJH.0b013e32832258c9

19. Bild DE, Jacobs DR, Liu K et al (1996) Seven-year trends in plasma low-density-lipoprotein-cholesterol in young adults: the CARDIA Study. Ann Epidemiol 6(3):235-245. https://doi.org/10.1016/10472797(96)00005-1

20. Warnick GR (1986) Enzymatic methods for quantification of lipoprotein lipids. Methods Enzymol 129:101-123

21. Friedewald WT, Levy RI, Fredrickson DS (1972) Estimation of the concentration of low-density lipoprotein cholesterol in plasma, without use of the preparative ultracentrifuge. Clin Chem 18(6): 499-502

22. Park K, Gross M, Lee DH et al (2009) Oxidative stress and insulin resistance: the Coronary Artery Risk Development in Young Adults Study. Diabetes Care 32(7):1302-1307. https://doi.org/10.2337/ dc09-0259

23. Bancks MP, Kershaw K, Carson AP, Gordon-Larsen P, Schreiner PJ, Carnethon MR (2017) Association of modifiable risk factors in young adulthood with racial disparity in incident type 2 diabetes during middle adulthood. JAMA 318(24):2457-2465. https://doi. org/10.1001/jama.2017.19546

24. Kilpatrick ES, Rigby AS, Atkin SL (2008) A1C variability and the risk of microvascular complications in type 1 diabetes: data from the Diabetes Control and Complications Trial. Diabetes Care 31(11):2198-2202. https://doi.org/10.2337/dc08-0864

25. Yano Y, Ning H, Allen N et al (2014) Long-term blood pressure variability throughout young adulthood and cognitive function in midlife: the Coronary Artery Risk Development in Young Adults (CARDIA) study. Hypertension 64(5):983-988. https://doi.org/10. 1161/HYPERTENSIONAHA.114.03978

26. Li T-C, Yang C-P, Tseng S-T et al (2017) Visit-to-visit variations in fasting plasma glucose and $\mathrm{HbA} 1 \mathrm{c}$ associated with an increased risk 
of Alzheimer disease: Taiwan Diabetes Study. Diabetes Care 40(9): 1210-1217. https://doi.org/10.2337/dc16-2238

27. Kim C, Sohn JH, Jang MU et al (2015) Association between visitto-visit glucose variability and cognitive function in aged type 2 diabetic patients: a cross-sectional study. PLoS One 10(7): e0132118. https://doi.org/10.1371/journal.pone.0132118

28. van Buuren S (2007) Multiple imputation of discrete and continuous data by fully conditional specification. Stat Methods Med Res 16(3):219-242. https://doi.org/10.1177/0962280206074463

29. Yuan YC (2000) Multiple Imputation for missing data: concepts and new development (version 9.0). In: Proceedings of the Twenty-Fifth Annual SAS Users Group International Conference. SAS Institute, Cary, NC, pp 1-11

30. Austin PC, Lee DS, Fine JP (2016) Introduction to the analysis of survival data in the presence of competing risks. Circulation 133(6): 601-609. https://doi.org/10.1161/CIRCULATIONAHA.115. 017719

31. Kim JA, Lee JS, Chung HS et al (2018) Impact of visit-to-visit fasting plasma glucose variability on the development of type 2 diabetes: a nationwide population-based cohort study. Diabetes Care 41(12):2610-2616. https://doi.org/10.2337/dc18-0802

32. Ghouse J, Skov MW, Kanters JK et al (2018) Visit-to-visit variability of hemoglobin A1c in people without diabetes and risk of major adverse cardiovascular events and all-cause mortality. Diabetes Care 42(1):134-141. https://doi.org/10.2337/dc18-1396
33. Monnier L, Mas E, Ginet C et al (2006) Activation of oxidative stress by acute glucose fluctuations compared with sustained chronic hyperglycemia in patients with type 2 diabetes. JAMA 295(14): 1681-1687. https://doi.org/10.1001/jama.295.14.1681

34. Keating ST, El-Osta A (2013) Glycemic memories and the epigenetic component of diabetic nephropathy. Curr Diab Rep 13(4): 574-581. https://doi.org/10.1007/s11892-013-0383-y

35. Diabetes Prevention Program Research Group (2002) Reduction in the incidence of type 2 diabetes with lifestyle intervention or metformin. N Engl J Med 346:393-403

36. Knowler WC, Fowler SE, Hamman RF et al (2009) 10-year followup of diabetes incidence and weight loss in the Diabetes Prevention Program Outcomes Study. Lancet 374(9702):1677-1686. https:// doi.org/10.1016/S0140-6736(09)61457-4

37. Echouffo-Tcheugui J, Zhao S, Brock G, Matsouaka R, Kline D, Joseph J (2019) Visit-to-visit glycemic variability and risks of cardiovascular events and all-cause mortality: the ALLHAT study. Diabetes Care 42(3):486-493. https://doi.org/10.2337/dc18-1430

Publisher's note Springer Nature remains neutral with regard to jurisdictional claims in published maps and institutional affiliations. 